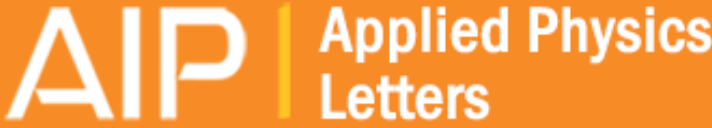

\section{Direct observation of nanometer-scale amorphous layers and oxide crystallites at grain boundaries in polycrystalline $\mathrm{Sr} 1-x \mathrm{~K} x$ Fe 2 As 2 superconductors}

Lei Wang, Yanwei Ma, Qingxiao Wang, Kun Li, Xixiang Zhang, Yanpeng Qi, Zhaoshun Gao, Xianping Zhang , Dongliang Wang, Chao Yao, and Chunlei Wang

Citation: Applied Physics Letters 98, 222504 (2011); doi: 10.1063/1.3592580

View online: http://dx.doi.org/10.1063/1.3592580

View Table of Contents: http://scitation.aip.org/content/aip/journal/apl/98/22? ver=pdfcov

Published by the AIP Publishing

\section{Articles you may be interested in}

Evidence for composition variations and impurity segregation at grain boundaries in high current-density polycrystalline $\mathrm{K}$ - and Co-doped BaFe2As2 superconductors

Appl. Phys. Lett. 105, 162604 (2014); 10.1063/1.4898191

Generic Fe buffer layers for Fe-based superconductors: Epitaxial FeSe1- x Te x thin films Appl. Phys. Lett. 99, 202503 (2011); 10.1063/1.3660257

Magnetic field dependent critical current density of $\mathrm{Bi}-\mathrm{Sr}-\mathrm{Ca}-\mathrm{Cu}-\mathrm{O}$ superconductor in bulk and tape form with addition of $\mathrm{Fe} 3 \mathrm{O} 4$ magnetic nanoparticles

J. Appl. Phys. 105, 07E311 (2009); 10.1063/1.3070628

Direct observation of nanometer-scale Mg- and B-oxide phases at grain boundaries in MgB 2

Appl. Phys. Lett. 79, 1837 (2001); 10.1063/1.1404127

Critical current in high T c grain boundary junctions

J. Appl. Phys. 84, 3972 (1998); 10.1063/1.368576

You don't

still use this

cell phone

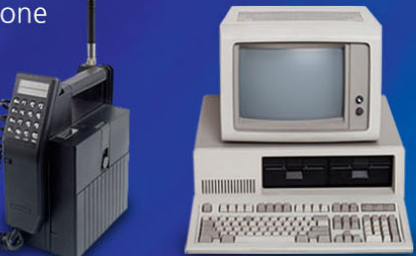

Why are you still using an AFM designed in the 80 's?
It is time to upgrade your AFM Minimum \$20,000 trade-in discount for purchases before August 31st

Asylum Research is today's technology leader in AFM 


\title{
Direct observation of nanometer-scale amorphous layers and oxide crystallites at grain boundaries in polycrystalline $\mathrm{Sr}_{1-\mathrm{x}} \mathrm{K}_{\mathrm{x}} \mathrm{Fe}_{2} \mathrm{As}_{2}$ superconductors
}

\author{
Lei Wang, ${ }^{1}$ Yanwei Ma, ${ }^{1, a)}$ Qingxiao Wang, ${ }^{2}$ Kun $\mathrm{Li}^{2}{ }^{2}$ Xixiang Zhang, ${ }^{2, \mathrm{a})}$ Yanpeng $\mathrm{Qi},{ }^{3}$ \\ Zhaoshun Gao, ${ }^{3}$ Xianping Zhang, ${ }^{3}$ Dongliang Wang, ${ }^{3}$ Chao Yao, ${ }^{3}$ and Chunlei Wang ${ }^{3}$ \\ ${ }^{1}$ Key Laboratory of Applied Superconductivity, Institute of Electrical Engineering, Chinese Academy \\ of Sciences, P.O. Box 2703, Beijing 100190, People's Republic of China \\ ${ }_{2}^{2}$ Imaging and Characterization Core Lab, King Abdullah University of Science and Technology, \\ Thuwal 23955-6900, Saudi Arabia \\ ${ }^{3}$ Key Laboratory of Applied Superconductivity, Institute of Electrical Engineering, Chinese Academy \\ of Sciences, P.O. Box 2703, Beijing 100190, People's Republic of China
}

(Received 23 March 2011; accepted 29 April 2011; published online 1 June 2011)

\begin{abstract}
We report here an atomic resolution study of the structure and composition of the grain boundaries in polycrystalline $\mathrm{Sr}_{0.6} \mathrm{~K}_{0.4} \mathrm{Fe}_{2} \mathrm{As}_{2}$ superconductor. A large fraction of grain boundaries contain amorphous layers larger than the coherence length, while some others contain nanometer-scale crystallites sandwiched in between amorphous layers. We also find that there is significant oxygen enrichment at the grain boundaries. Such results explain the relatively low transport critical current density $\left(J_{c}\right)$ of polycrystalline samples with respect to that of bicrystal films. (C) 2011 American Institute of Physics. [doi:10.1063/1.3592580]
\end{abstract}

The discovery of superconductivity in $\mathrm{Fe}-\mathrm{As}$ based compounds, with a critical transition temperature as high as $55 \mathrm{~K}$, has sparked enormous research effort to understand its structure-property relationships. ${ }^{1-6}$ One aspect of $\mathrm{Fe}-\mathrm{As}$ based superconductors that has particular technological importance is that the grain boundaries in bicrystals do not appear to lower the overall critical current $\left(J_{c}\right)$ as heavily as $\mathrm{YBa}_{2} \mathrm{Cu}_{3} \mathrm{O}_{7-\delta}(\mathrm{YBCO}){ }^{7,8} \mathrm{~A}$ remarkable transport intergrain $J_{c}$ of $\sim 10^{5} \mathrm{~A} / \mathrm{cm}^{2}$ at $4 \mathrm{~K}$ and self field has been reported in [001]-tilt cobalt-doped $\mathrm{BaFe}_{2} \mathrm{As}_{2}$ bicrystals with a high misorientation angle of $45^{\circ},{ }^{8}$ offering the possibility of a number of industrial applications. This is in contrast to polycrystalline samples, which show a relative low intergrain (transport) $J_{c}$ of $\sim 10^{3} \mathrm{~A} / \mathrm{cm}^{2}$ at $4.2 \mathrm{~K}$ and self field. ${ }^{3-5,9}$ The first critical step in understanding the mechanism by which polycrystalline samples cannot support a high transport $J_{c}$ is to determine the structure and composition of the grain boundaries.

In this letter, we report a series of atomic resolution studies of the grain boundaries in a polycrystalline $\mathrm{Sr}_{0.6} \mathrm{~K}_{0.4} \mathrm{Fe}_{2} \mathrm{As}_{2}$ sample. Three distinctive types of grain boundaries are found: clean grain boundaries, boundaries containing amorphous layer with a width of $\sim 10 \mathrm{~nm}$ (i.e., larger than the coherence length), and boundaries containing amorphous-crystallite-amorphous trilayers $\sim 30 \mathrm{~nm}$ in width. Furthermore, there is significant oxygen enrichment in the amorphous layer and crystallites. Such boundary features indicate that a large fraction of grain boundaries act as critical current barriers, and that control of oxygen content at grain boundaries will be essential for attaining optimal polycrystalline critical currents.

The polycrystalline $\mathrm{Sr}_{0.6} \mathrm{~K}_{0.4} \mathrm{Fe}_{2} \mathrm{As}_{2}$ samples used in this study were synthesized by one-step solid state reaction, which produces a sharp critical temperature $\left(T_{c}\right)$ transition at

\footnotetext{
${ }^{\text {a) }}$ Authors to whom correspondence should be addressed. Electronic addresses: ywma@mail.iee.ac.cn and xixiang.zhang@kaust.edu.sa.
}

$\sim 35 \mathrm{~K}$ and a quasisingle phase (for a more detailed description of the sample preparation see Ref. 10). One way to directly observe structure, composition, and bonding effects at grain boundaries is through the combination of highresolution transmission electron microscopy (TEM) and electron energy loss spectroscopy (EELS) at atomic resolution in a scanning TEM (STEM). Our experiments here use an FEI Titan 80-300 kV (ST) STEM operated at $300 \mathrm{kV}$ which has a point resolution of $\sim 0.205 \mathrm{~nm}$ in TEM mode and a $0.14 \mathrm{~nm}$ STEM resolution. Under this experimental
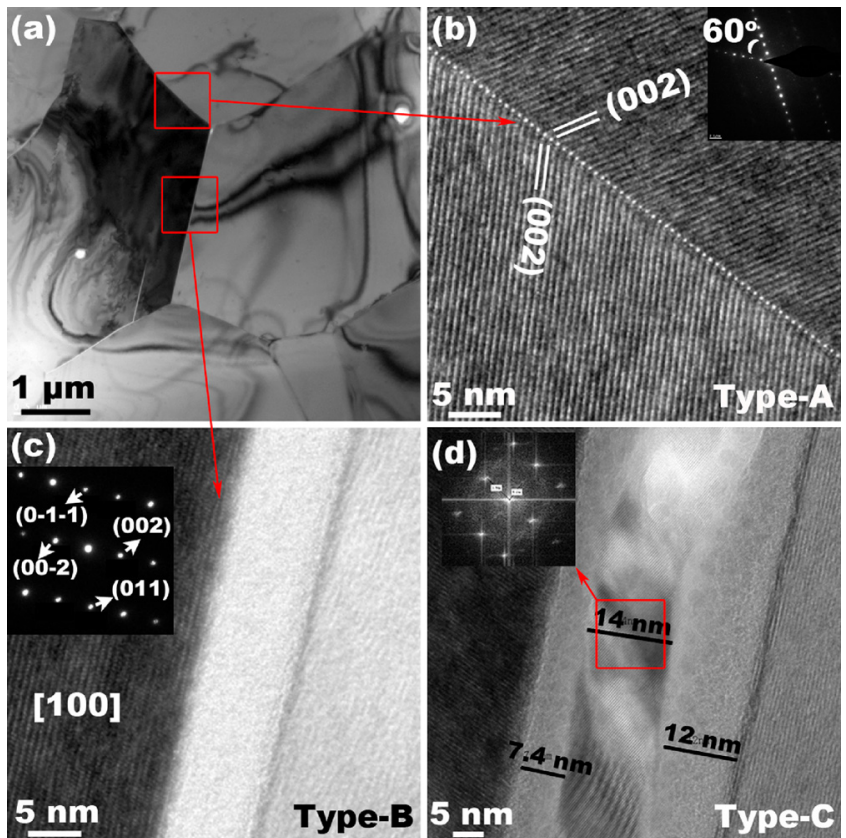

FIG. 1. (Color online) (a) TEM image of an apparently well connected grain boundary network in polycrystalline $\mathrm{Sr}_{0.6} \mathrm{~K}_{0.4} \mathrm{Fe}_{2} \mathrm{As}_{2}$. (b) A high-resolution TEM image of a typical, clean high-angle grain boundary. (c) The detailed structure of a grain boundary containing an amorphous layer about $10 \mathrm{~nm}$ in thickness. (d) Another type of grain boundary containing nanometer-scale impurity crystallites sandwiched between amorphous layers. 
(a)
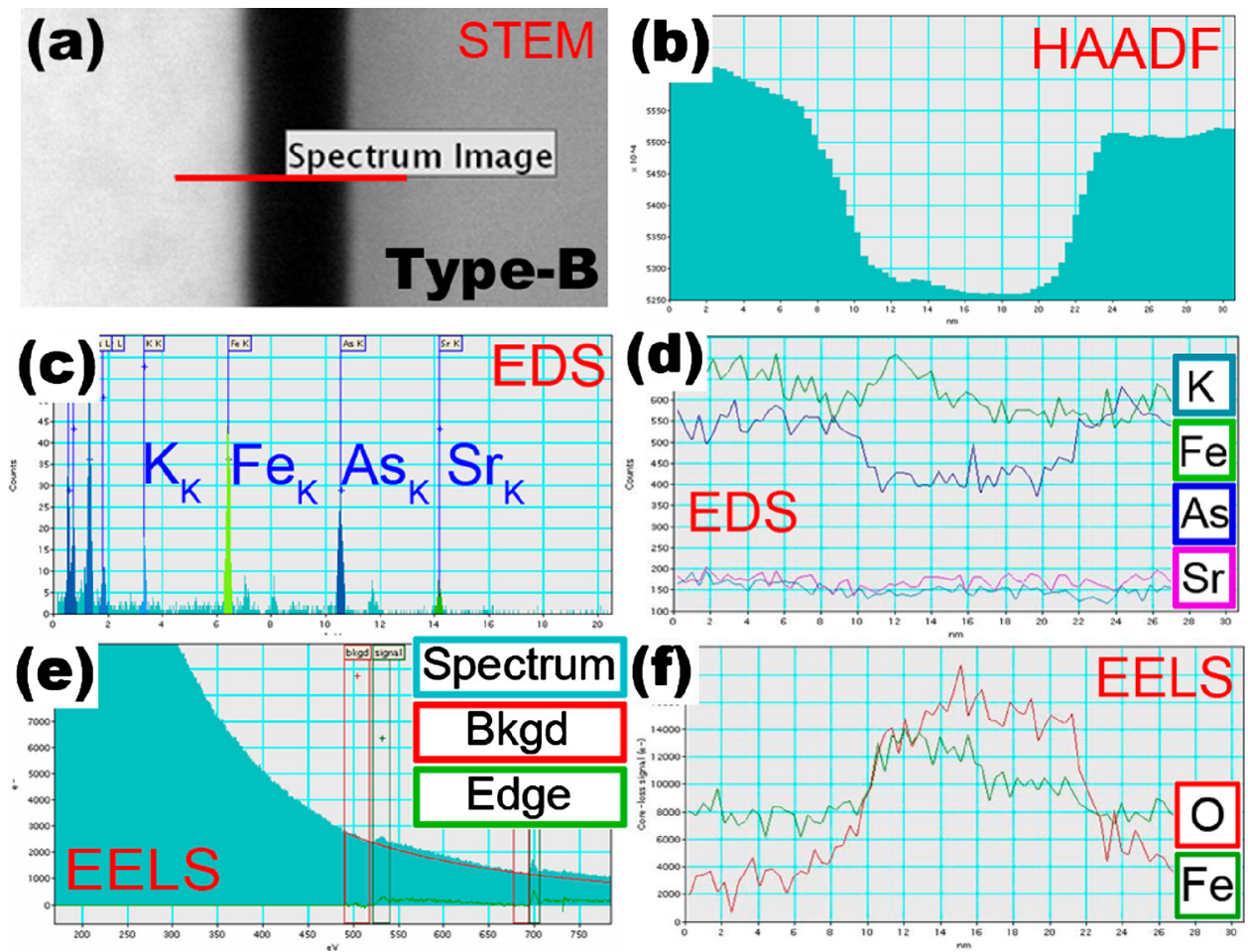

FIG. 2. (Color online) (a) A STEM image of the type-B grain boundary. (b) HAADF-line scan across the grain boundary. [(c) and (d)] EDS spectra and line scan performed on the amorphous layer. [(e) and (f)] EELS spectra and line scan performed on the amorphous layer. setup, the high-resolution grain boundaries structure can be observed directly at TEM mode, while STEM imaging can be used to position the electron probe for EELS.

Figure 1(a) shows a grain boundary network in polycrystalline $\mathrm{Sr}_{0.6} \mathrm{~K}_{0.4} \mathrm{Fe}_{2} \mathrm{As}_{2}$ at low magnification. The $\mathrm{Sr}_{0.6} \mathrm{~K}_{0.4} \mathrm{Fe}_{2} \mathrm{As}_{2}$ grains, with typical grain sizes around $5 \mu \mathrm{m}$, appear to be well connected. Figure 1(b) shows a high-resolution TEM image of a typical, clean high-angle grain boundary (type-A), in which the sample was tilted so that the grain boundary was almost parallel to the incident electron beam. Only the (002) $\mathrm{Sr}_{0.6} \mathrm{~K}_{0.4} \mathrm{Fe}_{2} \mathrm{As}_{2}$ planes $\left(\mathrm{d}_{002}\right.$ $=0.658 \mathrm{~nm})$ are resolved in the both grains. The selected area diffraction pattern of the across section of the grain boundary region is shown in the inset of Fig. 1(b). The c-axis of one $\mathrm{Sr}_{0.6} \mathrm{~K}_{0.4} \mathrm{Fe}_{2} \mathrm{As}_{2}$ grain is rotated by about $60^{\circ}$ with respect to that of the other.
It is interesting to note that some apparently well connected grains are not actually well connected. Figure 1(c) shows the detailed structure of a grain boundary between two apparently well connected grains. It contains an amorphous layer of about $10 \mathrm{~nm}$ in thickness (type-B), which is lager than the coherence length $\sim 1-2 \mathrm{~nm},{ }^{11,12}$ consequently, resulting in a current blocking effect. Similar amorphous layers around individual grains have been reported in $\mathrm{NdFeAsO}_{1-\delta}$ and $\mathrm{Sr}_{0.6} \mathrm{~K}_{0.4} \mathrm{Fe}_{2} \mathrm{As}_{2} .{ }^{13,14}$ Another type of grain boundary labeled type-C contains nanometer-scale crystallites sandwiched between amorphous layers, as shown in Fig. 1(d). The nanometer-scale crystallites are confirmed to be an impurity phase from the selected area diffraction pattern [inset of Fig. 1(d)].

Figures 2(b) and 3(b) show high-angle annular dark field (HAADF) line scans across type-B [Fig. 2(a)] and type-C
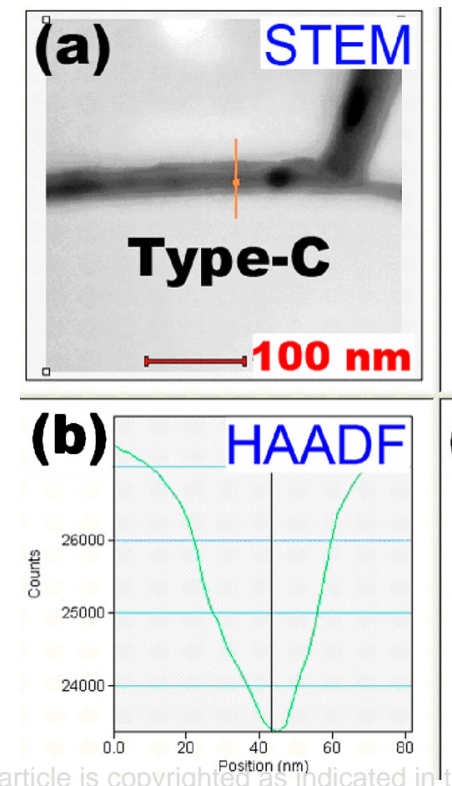
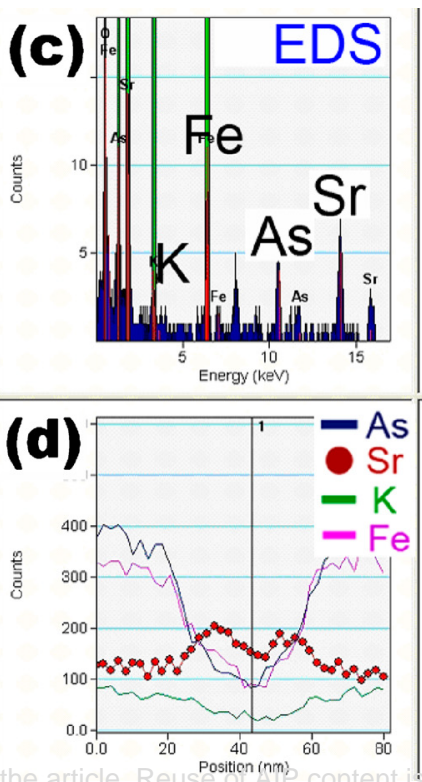
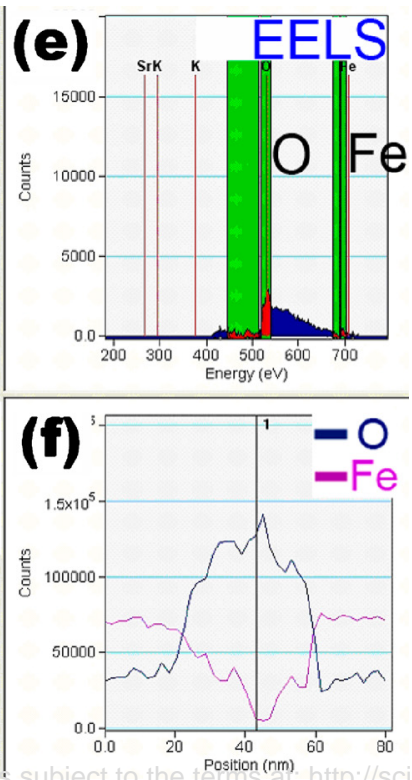

FIG. 3. (Color online) (a) A STEM image of the type- $\mathrm{C}$ grain boundary. (b) HAADF-line scan across the grain boundary. (c) EDS spectra and line scan performed on the amorphous-crystallite-amorphous trilayer. (e) EELS spectra and line scan performed on the amorphouscrystallite-amorphous trilayer. 


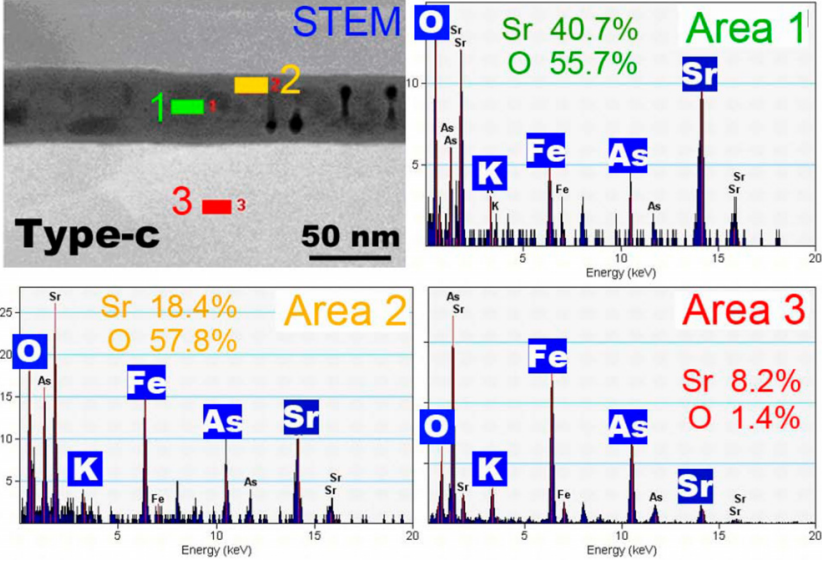

FIG. 4. (Color online) EDS analysis on the crystallite (area 1), amorphous layer (area 2) and grain (area 3)

[Fig. 3(a)] grain boundaries, respectively. The dimension of the grain boundaries determined by HAADF-line scans are consistent with that obtain from high-resolution TEM images. Energy dispersive x-ray spectroscopy (EDS)- and EELS-line scans have been performed simultaneously across the grain boundaries to analyze the composition of amorphous layers and the unknown crystallites. Peaks of $\mathrm{Sr}, \mathrm{K}$, $\mathrm{Fe}$, As, and $\mathrm{O}$ have been detected by EDS, as shown in Figs. 2(c) and 3(c). The ratio $\mathrm{Sr}: \mathrm{K}: \mathrm{Fe}: \mathrm{As}$ in grain is 0.55:0.44:2.0:2.0, nicely consistent with nominal composition. The composition variations across the type- $\mathrm{B}$ grain boundary are shown in Fig. 2(d), which shows that the amorphous layer do not contain as much level of arsenic as in grains. To determine the oxygen and iron contents precisely, EELS-line scan was also performed. A very small probe size $(0.3 \mathrm{~nm})$ and a pixel step of $2 \mathrm{~nm}$ have been used to record the oxygen $\mathrm{K}$, iron L edge [Fig. 2(e)]. The corresponding composition variations strongly suggest that oxygen at the region of type-B grain boundary is very high [Fig. 2(f)].

A different situation is found at type-C grain boundaries where nanometer-scale crystallites are sandwiched between amorphous layers. Similar to the type-B boundary described above, a low level of arsenic as well as a high level of oxygen is observed at the boundaries. However, as can be seen from the EDS- and EELS-line scan spectra in Fig. 3(d), the composition of amorphous layers in type- $\mathrm{C}$ grain boundaries are not consistent with that of type-B grain boundaries. It shows a significant enrichment of strontium in the amorphous layers in type-C grain boundaries. The crystallites sandwiched between amorphous layers show enormous changes in the EELS spectra compared to the grain features. Here, the iron L-edge intensity is reduced dramatically and the oxygen K-edge is increased by a large amount [Fig. 3(f)]. It should be mentioned that the Sr peak seems drop a little bit but $\mathrm{Fe}$ and As peak peaks drops more, so the $\mathrm{Sr}$ relative concentration is higher.

EDS analyses have been performed on the crystallite (area 1), amorphous layer (area 2), and grain (area 3), as shown in Fig. 4. As the EDS signal counts, especially for O, is not enough for accurate quantification, the data here just want to show that the $\mathrm{Sr}$ and $\mathrm{O}$ are really high in the area 1 and 2. According to TEM image of the unknown crystallites, d-space like $6.5 \AA$, $3.5 \AA, 3 \AA, 2.6 \AA$, and $2 \AA$ has been found. According to the $\mathrm{x}$-ray diffraction database, the structure of the unknown crystallites may be close to one of the tetragonal $\mathrm{SrO}_{2}$ structure. In area 3 which is far from the grain boundary, the $\mathrm{O}$ is only $1.4 \%$. This means the main phase of the material is still $\mathrm{Sr}_{0.6} \mathrm{~K}_{0.4} \mathrm{Fe}_{2} \mathrm{As}_{2}$ and $\mathrm{O}$ only exist close to the grain boundary.

The results described above clearly show that the grain boundaries in $\mathrm{Sr}_{0.6} \mathrm{~K}_{0.4} \mathrm{Fe}_{2} \mathrm{As}_{2}$ exhibit a propensity for oxygen enrichment. At this stage it is not clear whether the two different types of dirty boundaries we observed are fundamentally different or simply different stages of oxidation. While, it is immediately clear that type- $\mathrm{B}$ and type-C grain boundaries act as intergrain (transport) critical current barriers, and by itself may be enough to explain the relatively low transport critical current density $\left(J_{c}\right)$ of polycrystalline samples with respect to that of bicrystal films. It also indicates that control of oxygen content at grain boundaries will be essential for attaining optimal polycrystalline critical currents.

Authors are indebted to H. H. Wen, R. Flukiger, H. Kumakura, S. X. Dou, and T. Matsushita for useful comments. This work is partially supported by the National "973" Program (Grant No. 2011CBA00105), National Science Foundation of China (Grant No. 51025726), and Beijing Municipal Science and Technology Commission under Grant No. Z09010300820907.

${ }^{1}$ Y. Kamihara, T. Watanabe, M. Hirano, and H. Hosono, J. Am. Chem. Soc. 130, 3296 (2008).

${ }^{2}$ Z. Ren, Y.-Q. Wu, and L.-C. Zhang, Chin. Phys. Lett. 25, 2385 (2008).

${ }^{3}$ M. Rotter, M. Tegel, and D. Johrendt, Phys. Rev. Lett. 101, 107006 (2008)

${ }^{4}$ A. Yamamoto, A. A. Polyanskii, J. Jiang, F. Kametani, C. Tarantini, F. Hunte, J. Jaroszynski, E. E. Hellstrom, P. J. Lee, A. Gurevich, and D. C. Larbalestier, Supercond. Sci. Technol. 21, 095008 (2008).

${ }^{5}$ L. Wang, Z. S. Gao, Y. P. Qi, X. P. Zhang, D. L. Wang, and Y. W. Ma, Supercond. Sci. Technol. 22, 015019 (2009).

${ }^{6}$ E. S. Otabe, M. Kiuchi, S. Kawai, Y. Morita, J. Ge, B. Ni, Z. Gao, L. Wang, Y. Qi, X. Zhang, and Y. Ma, Physica C 469, 1940 (2009).

${ }^{7}$ S. Lee, J. Jiang, J. D. Weiss, C. M. Folkman, C. W. Bark, C. Tarantini, A. Xu, D. Abraimov, A. Polyanskii, C. T. Nelson, Y. Zhang, S. H. Baek, H. W. Jang, A. Yamamoto, F. Kametani, X. Q. Pan, E. E. Hellstrom, A. Gurevich, C. B. Eom, and D. C. Larbalestier, Appl. Phys. Lett. 95, 212505 (2009).

${ }^{8}$ T. Katase, Y. Ishimaru, A. Tsukamoto, H. Hiramatsu, T. Kamiya, K. Tanabe, and H. Hosono, arXiv:1011.5721v1 (unpublished).

${ }^{9}$ L. Wang, Y. P. Qi, D. L. Wang, X. P. Zhang, Z. S. Gao, Z. Y. Zhang, Y. W. Ma, S. Awaji, G. Nishijima, and K. Watanabe, Physica C 470, 183 (2010).

${ }^{10}$ Y. W. Ma, Z. S. Gao, Y. P. Qi, X. P. Zhang, L. Wang, Z. Y. Zhang, and D. L. Wang, Physica C 469, 651 (2009).

${ }^{11}$ J. Jaroszynski, F. Hunte, L. Balicas, Y. J. Jo, I. Raicevic, A. Gurevich, D. C. Larbalestier, F. F. Balakirev, L. Fang, P. Cheng, Y. Jia, and H. H. Wen, Phys. Rev. B 78, 174523 (2008).

${ }^{12}$ M. M. Altarawneh, K. Collar, C. H. Mielke, N. Ni, S. L. Bud'ko, and P.C. Canfield, Phys. Rev. B 78, 220505(R) (2008).

${ }^{13}$ J. D. Moore, K. Morrison, K. A. Yates, A. D. Caplin, Y. Yeshurun, L. F. Cohen, J. M. Perkins, C. M. McGilvery, D. W. McComb, Z. A. Ren, J. Yang, W. Lu, X. L. Dong, and Z. X. Zhao, Supercond. Sci. Technol. 21, 092004 (2008).

${ }^{14}$ L. Wang, Y. P. Qi, X. P. Zhang, Z. S. Gao, D. L. Wang, and Y. W. Ma, Supercond. Sci. Technol. 23, 025027 (2010). 